\title{
Phylogenetic analyses and species delimitation in the section Corydalis (Papaveraceae) based on chloroplast markers
}

\author{
A. $\mathrm{Oh}$ \\ Department of Biology, Chungbuk National University, Cheongju, South \\ Korea \\ Corresponding author: A. Oh \\ E-mail: ohamiohami@gmail.com
}

Genet. Mol. Res. 21 (1): gmr18996

Received November 30, 2021

Accepted December 21, 2021

Published January 31, 2022

DOI http://dx.doi.org/10.4238/gmr18996

\begin{abstract}
The genus Corydalis (Papaveraceae), which is distributed in temperate regions of the northern hemisphere, has been taxonomically studied mainly on the basis of morphological and molecular genomic information. In the present study, 14 species that belong to the Korean section Corydalis were collected in South Korea and phylogenetically analyzed using four chloroplast genomic regions, which include $m a t K, r b c L, r p L 16$ genes and the $\operatorname{trn} G$ intron. The author tried to include the nuclear Internal Transcribed Spacer (ITS) region in the phylogenetic analysis; however, multiple PCR bands and various band sizes observed led to the conclusion that the ITS region is not suitable for the phylogenetic study of Corydalis. When the four chloroplast genomic regions were separately analyzed, different levels of resolution for species delimitation were observed, and in most cases the resolution levels were quite low. When matK and $r p L 16$ were concatenated, the highest resolution for species delimitation was observed. However, when other regions were added to this concatenated region to improve the resolution, the resolution decreased, which was in contrast to the author's expectation and deserves further analysis. At the same time, the author observed inconsistencies between the previously established taxonomy based on morphology and the molecular phylogenies in the present study. This discrepancy needs to be addressed in further detail, so that the
\end{abstract}


taxonomy of the genus Corydalis can fully incorporate both morphology and molecular genomic information. Overall, the present study provides insights into the taxonomy of Corydalis, and clearly demonstrates that proper combinations of chloroplast markers can lead to successful discrimination of the species in this genus. Indeed, this study suggests ways to better utilize phylogenetic analyses and species delimitation in this interesting and complicated taxon. Since Corydalis is taxonomically challenging and widely used as medicinal plants in Asia, this study can be a valuable source of information on this genus.

Key words: Chloroplast genome; Corydalis; Phylogeny; Species delimitation; Taxonomy

\section{INTRODUCTION}

The genus Corydalis DC., which belongs to the family Papaveraceae, consists of about 440-465 species worldwide (Liden, 1996; Zhang et al., 2008). This genus can be distinguished from other genera within the same family on the basis of various morphological characteristics, including symmetrical flowers, racemose inflorescence, and seeds with elaiosomes. Corydalis is distributed in temperate regions of the northern hemisphere, including northeastern Asia and the Himalayas (Oh, 1999; Oh et al., 2004; Ren et al., 2018). Notably, 357 species and 262 endemics belonging to this genus are found in China (Zhang et al., 2008). Liden (1996) reported that Chinese and Tibetan regions are the centers of distribution for this genus based on the fact that all sections of Corydalis are present in these regions.

Since De Candolle described the genus Corydalis for the first time in 1805, Hooker and Thomson (1855), Fedde (1936), Liden (1996), and other researchers have conducted taxonomic studies on it. Corydalis was considered to belong to the family Fumariaceae by some researchers (Lidén, 1996; Oh, 1999; Oh et al., 2004; Oh et al., 2010). However, recently, Papaveraceae is more widely recognized as the family of Corydalis (Jiang et al., 2018; Ren et al., 2019; Li et al., 2020; Xu and Wang, 2021). Palibin (1898) first studied Korean Corydalis species, and subsequently, Komarov (1903), Nakai (1909, 1914, 1952), and other taxonomists recorded different numbers of species, varieties and formae for this genus. Oh (1986) conducted comprehensive research on Korean Corydalis and reported that the genus Corydalis consists of four sections: section Corydalis, section Duplotuber, section Ramoso-sibiricae, and section Sophorocapnos (Oh, 1999). However, although ample studies have been conducted on the genus Corydalis, it is generally recognized that its taxonomy is difficult to elucidate because of its complicated and diverse morphological characters (Ren et al., 2018; Xu and Wang, 2021). Some phylogenetic studies which used different molecular markers were conducted to understand the taxonomy of Corydalis (Liden et al., 1995; 1997), but accurate species-level systematics remains incomplete. Species delimitation using the phylogenetic approach is a critical issue in understanding the taxonomy of Corydalis, and needs to be further explored. 
In phylogenetic studies, some genetic markers are globally used across many different taxa. The Internal Transcribed Spacer (ITS) region of nuclear ribosomal DNA is one of the most popular nuclear markers that is considered to be a useful barcode in plant systematics. It is generally known that ITS can be used to reveal low-level taxonomy, including the relationships between close genera or species within the same genus (Yuan et al., 1996; Alvarez and Wendel, 2003; Mort et al., 2007). Chloroplast genome sequences are also commonly used markers for plant phylogenetic studies (Shaw et al., 2005; Mort et al., 2007; Dong et al., 2012). In addition, recently, complete chloroplast genome sequences are being much investigated as well for the taxonomy and the phylogenetic analyses of the target plants (Wu et al., 2020; Liu et al., 2021). In previous phylogenetic studies on Corydalis, ITS and chloroplast markers have been used and their value as DNA barcodes has been assessed (Jiang et al., 2018; Ren et al., 2019).

In the present study, the author provides phylogenetic relationships within the Korean section Corydalis using four chloroplast genome sequences, which are matK, $r b c L, r p L 16$ genes and the $\operatorname{trn} G$ intron. By generating phylogenies within section Corydalis, the author aimed to enhance the general understanding for the taxonomy of this taxon and to unravel whether the markers used here are useful tools for species delimitation in section Corydalis. In addition, the author aimed to explain the inconsistency between the phylogenetic analysis in this study and previously established taxonomy, which is based on morphology. As some markers used in the present study have been previously used in the phylogenetic studies on Corydalis, the author expected to effectively complement previous studies on the same taxon as well. Given that chloroplast markers are generally used in phylogenetic studies, this study can address the important and fundamental questions on the utility of chloroplast. Moreover, since Corydalis is widely used as medicinal plants in Asian regions, the present study which focuses on species delimitation can greatly contribute to the effective use of this genus.

\section{MATERIAL AND METHODS}

\section{Sample collection}

All 32 samples used in this study were collected from South Korea (Table 1). Thirteen clearly identified species and one unidentified taxon (Corydalis sp.) belonging to the section Corydalis were analyzed. Two to three individuals from different locations were used for each species. In the case of Corydalis alata, only one individual was used. Corydalis heterocarpa and Corydalis speciosa, which belong to the section Sophorocapnos, were designated as outgroups. The previously established taxonomic classifications used as references were generally based on morphological information.

Genomic DNA was extracted from leaf tissues using the DNeasy Mini Kit (QIAGEN, Seoul, Korea). When a fresh leaf was not available, a dried leaf from a voucher specimen was used instead; $100 \mathrm{mg}$ of fresh leaf tissue and $20 \mathrm{mg}$ of dried leaf 
tissue from the voucher specimens were used for the extraction. The protocol provided by the manufacturer was used for DNA extraction.

Table 1. Species used in this study of the section Corydalis and the sampling sites in South Korea.

\begin{tabular}{|c|c|}
\hline Specie & Site \\
\hline Corydalis albipetala & Gapyeong county, Nojeok mountaintop \\
\hline Corydalis albipetala & Pyeongchang county, Woljeong temple \\
\hline Corydalis albipetala & Pyeongchang county, Woljeong temple entrance \\
\hline Corydalis turtschaninovii & Yeongju city, Geumgye lake \\
\hline Corydalis turtschaninovii & Wonju city, Anchang town \\
\hline Corydalis humilis & Yeongju city, Sobaek mountain, Choam temple \\
\hline Corydalis humilis & Namyangju city, Chukryeong mountain \\
\hline Corydalis namdoensis & Yeongcheon city, Bohyeon mountain \\
\hline Corydalis namdoensis & Kyeongju city, Toham mountain \\
\hline Corydalis remota & Hapcheon county, Odo mountain \\
\hline Corydalis remota & Cheongyang county, Chilgap mountain, Chilgap large bridge \\
\hline Corydalis lineariloba & Taebaek city, Baekdan temple \\
\hline Corydalis lineariloba & Jeongseon county, Hambaek mountain \\
\hline Corydalis hallaisanensis & Seoguipo city, 1100 highland \\
\hline Corydalis hallaisanensis & Jeju city, Sangumburi crater \\
\hline Corydalis alata & Donghae city, Chorok mountaintop \\
\hline Corydalis maculata & Namyangju city, Cheonma mountain \\
\hline Corydalis maculata & Bonghwa county, Cheongryang mountain \\
\hline Corydalis cornupetala & Kyeongsan city, Kyejeong forest \\
\hline Corydalis cornupetala & Kyeongsan city, Kyejeong forest 2 \\
\hline Corydalis filistipes & Wooloong county, Hongmun town \\
\hline Corydalis filistipes & Wooloong county, intake station \\
\hline Corydalis grandicalyx & Wonju city, Soyongsodong valley \\
\hline Corydalis grandicalyx & Gapyeong county, Ajaebi hill \\
\hline Corydalis grandicalyx & Yangpyeong county, Nojeok mountaintop \\
\hline Corydalis hirtipes & Inje county, Yongdae town, Majang space 1 \\
\hline Corydalis hirtipes & Inje county, Yongdae town, Majang space 2 \\
\hline Corydalis sp. & Gongju city, Gyeryong mountain, Donghak temple \\
\hline Corydalis sp. & Jangseong county, Naejang mountain, Gain town \\
\hline Corydalis sp. & Soonchang county, Gangcheon mountain \\
\hline Corydalis heterocarpa & Gongju city, Gyeryong mountain, Gap temple \\
\hline Corydalis speciosa & Eumseong county, Gaseop mountain \\
\hline
\end{tabular}

To perform PCR amplification of five genomic regions, namely the ITS region in the nucleus and $m a t K, r b c L, r p L 16$, and $\operatorname{trn} G$ intron in the chloroplast, five previously reported primer pairs were used. The primer sequences are provided in Table 2. PCR was performed using the ABI ProFlex PCR System (Applied Biosystems, Seoul, Korea) in a $25 \mu \mathrm{L}$ mixture consisting of $2 \mu \mathrm{L}$ template DNA, $2.5 \mu \mathrm{L} 10 \mathrm{X}$ buffer, $2 \mu \mathrm{L}$ dNTP, $1 \mu \mathrm{L}$ primer, $0.25 \mu \mathrm{L}$ IP-pro-taq, and $17.25 \mu \mathrm{L}$ DW. The same PCR conditions were used for all five genomic regions. The PCR conditions were as follows: initial denaturation at $94^{\circ} \mathrm{C}$ for 4 min followed by 35 cycles of denaturation at $94^{\circ} \mathrm{C}$ for $30 \mathrm{sec}$, annealing at $53^{\circ} \mathrm{C}$ for $30 \mathrm{sec}$, extension at $72^{\circ} \mathrm{C}$ for $1 \mathrm{~min} 30 \mathrm{sec}$, and final extension at $72^{\circ} \mathrm{C}$ for $10 \mathrm{~min}$.

Sequencing was performed in both directions using the ABI 3730xl Analyzer (Applied Biosystems, Seoul, Korea) and the primers used for sequencing were the same as those used for PCR amplification. The generated sequence data were assembled using SeqMan ${ }^{\circledR}$ (DNASTAR) software. PCR amplification of the nuclear ITS region 
did not generate meaningful results that could be further analyzed. Therefore, only the four chloroplast genomic regions were sequenced.

\begin{tabular}{|c|c|c|c|}
\hline Region & Primer & Sequences & References \\
\hline nrITS & $\begin{array}{l}\text { ITS5 } \\
\text { ITS4 }\end{array}$ & $\begin{array}{l}\text { GGAAGTAAAAGTCGTAACAAGG } \\
\text { TCCTCCGCTTATTGATATGC }\end{array}$ & White et al. 1990 \\
\hline matK & $\begin{array}{l}\text { matK1166 } \\
\text { matK192 }\end{array}$ & $\begin{array}{l}\text { GGCTTACTAATGGGAT } \\
\text { CGGGTTGCAAMAATAAAGGA }\end{array}$ & $\begin{array}{l}\text { Perez-Gutierrez et al. } \\
2015\end{array}$ \\
\hline$r p L 16$ & $\begin{array}{l}\text { rpL16F71 } \\
\text { rpL16R1516 }\end{array}$ & $\begin{array}{l}\text { GCTATGCTTAGTGTGTGACTCGTTG } \\
\text { CCCTTCATTCTTCCTCTATGTTG }\end{array}$ & Shaw et al. 2005 \\
\hline $\operatorname{trn} G$ intron & $\begin{array}{l}\text { 3'trnG } \\
\text { 5'trnG2G }\end{array}$ & $\begin{array}{l}\text { GTAGCGGGAATCGAACCCGCATC } \\
\text { GCGGGTATAGTTTAGTGGTAAAA }\end{array}$ & Shaw et al. 2005 \\
\hline$r b c L$ & $\begin{array}{l}r b c L-1 \mathrm{~F} \\
r b c L-724 \mathrm{R}\end{array}$ & $\begin{array}{l}\text { ATGTCACCACAAACAGAAAC } \\
\text { TCGCATGTACCTGCAGTAGC }\end{array}$ & Kress et al., 2005 \\
\hline
\end{tabular}

\section{Phylogenetic analysis}

The sequences were aligned using MUSCLE (Edgar, 2004) implemented in MEGA7 (Kumar et al., 2016) software. Phylogenetic trees were constructed using MEGA7, the same software. The four chloroplast genomic regions were analyzed separately or in combination. Maximum Likelihood (ML) approach was used for phylogenetic analyses.

\section{RESULTS}

When PCR amplification of the nuclear ITS region was performed, more than one band were observed in many cases, and the sizes of these bands were highly variable. Therefore, this region could not be used for phylogenetic analyses. The observation of unexpected PCR band numbers and sizes in the ITS region led to the conclusion that this region is not appropriate for the phylogenetic study of the genus Corydalis. When matK, $r b c L, r p L 16$, and $\operatorname{trn} G$ intron regions were separately used for phylogenetic analyses, many species belonging to the section Corydalis did not show monophyletic clustering, indicating that these genomic regions are not suitable for discriminating species when used alone. When the matK region was used alone for phylogenetic analysis, Corydalis turtschaninovii, Corydalis hirtipes, Corydalis grandicalyx, Corydalis albipetala, and Corydalis filistipes showed monophyly each, but other species were not successfully delimited. The $r p L 16$ region did not have sufficient resolution for discriminating species, except for $C$. turtschaninovii, $C$. hirtipes, $C$. filistipes, and $C$. grandicalyx. In $r b c L$ and $\operatorname{trn} G$ intron, taxonomically meaningful clustering patterns that could identify individual species were not observed except in the case of two or three species. However, when matK and $r p L 16$ were used together, the resolution for species discrimination increased and the highest number of Corydalis species, i.e., six species including $C$. turtschaninovii, $C$. hirtipes, $C$. grandicalyx, $C$. filistipes, C. albipetala, and C. cornupetala, were distinguished (Figure 1). In this case, monophyly of five out of six species was supported by $99 \%$ bootstrap value. 
Interestingly, when other regions, which are $r b c L$ and $\operatorname{trn} G$ intron, were added to these two concatenated regions, the resolution for species delimitation decreased, resulting in five species being discriminated (Figure 2). In this case, monophyly of four out of five species was supported by $100 \%$ bootstrap value.

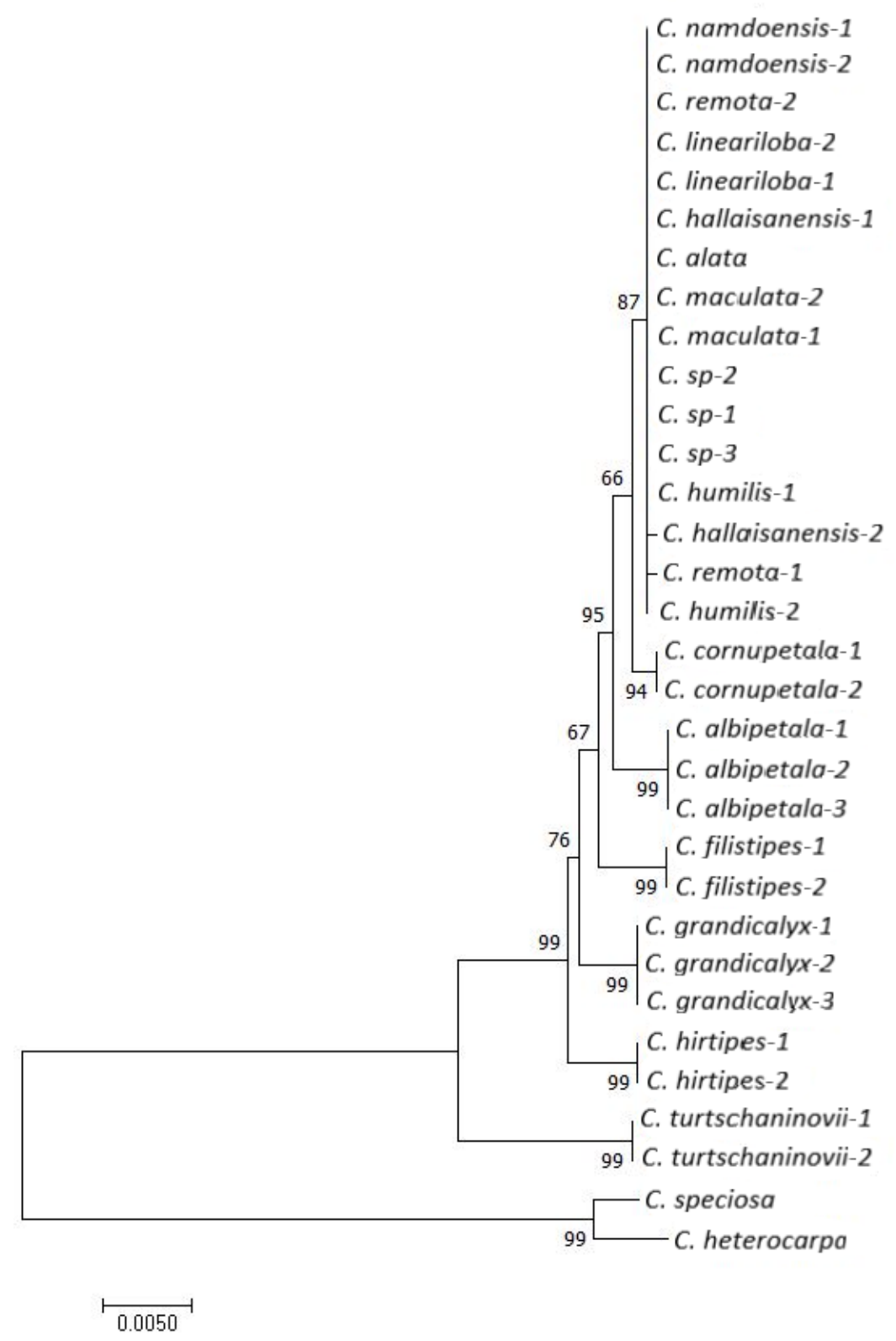

Figure 1. Maximum Likelihood phylogenetic tree of the Korean section Corydalis based on concatenated matK and $r p L 16$. 


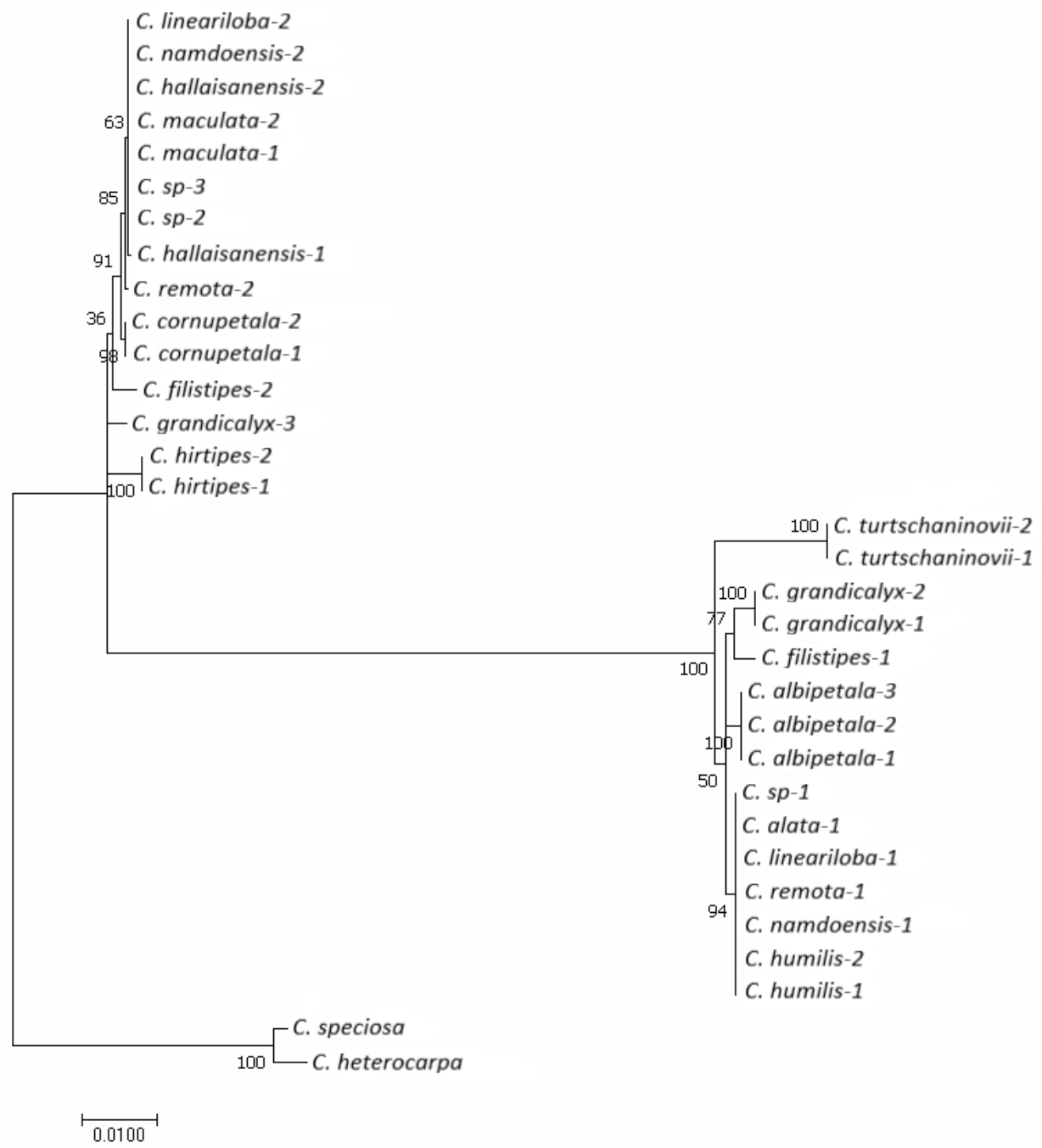

Figure 2. Maximum Likelihood phylogenetic tree of the Korean section Corydalis based on concatenated matK, $r p L 16, r b c L$ and $\operatorname{trn} G$ intron.

\section{DISCUSSION}

The ITS region of nuclear ribosomal DNA has been often used in phylogenetic studies of the genus Corydalis (Liden et al., 1995; Jiang et al., 2018; Ren et al., 2018; Li et al., 2020). While some of these studies supported the general efficacy of nrITS in the taxonomic analysis of Corydalis, other doubted about its efficacy. Jiang et al. (2018) reported "the multi-copy nature of ITS region in Corydalis" for the first time and suggested that research on Corydalis using the ITS region needs to be conducted with caution, even though their study could utilize ITS by conducting cloning. The report of Jiang et al. is consistent with this result, where multiple PCR bands were observed and 
further phylogenetic analysis was impossible. One unpublished study previously tried using ITS in phylogenetic study as well, but PCR amplification resulted in multiple bands and various band sizes (data not shown), which is very similar to the result of the present study. Through the author's analysis, the author clarified that the ITS region is not as useful as generally expected, at least in the case of genus Corydalis. The findings effectively complemented previous reports on the similar observations.

On performing phylogenetic analyses using four chloroplast genomic regions, the author found that some Corydalis species, such as $C$. turtschaninovii, $C$. hirtipes, $C$. filistipes, and $C$. grandicalyx, could be clearly delimited with one or two chloroplast markers. This supports previously established species delimitations based on morphological information. At the same time, the author found that when these chloroplast markers were used alone, the resolution for species discrimination in Corydalis was quite low. When different genomic regions were concatenated, the resolution increased to some extent, but not much. When all four regions were used together, the resolution was lower than that when matK and $r p L 16$ were concatenated, which was in contrast to the initial expectation. These indicate that sequence variations in the chloroplast genomic regions used by the author are rather low and that concatenating many regions does not necessarily lead to higher species discrimination resolution. In general, it is known that concatenation of molecular markers results in higher resolution for classification (Bapteste et al., 2008), and many previous studies have used this concatenation approach (Amrine-Madsen et al., 2003; Rokas et al., 2003; Hedges et al., 2004). However, the concatenation of markers can be problematic and may not necessarily lead to the high resolution power the author expect in phylogenetic studies (Kubatko and Degnan, 2007). Specifically, studies have suggested that concatenating many genes in phylogenetic analyses can lead to failure because of the differences in the evolutionary histories of individual genes (Kolaczkowski and Thornton, 2004; Mossel and Vigoda, 2005; Kubatko and Degnan, 2007). Notably, the observation of decreased resolution level deserves further analyses because these analyses can contribute to understanding how genomic markers reflect the actual systematics of target plants and can provide valuable information on how to combine many genomic regions in the phylogenetic study when trying to clearly identify the taxonomy. Ultimately, these analyses may increase the efficiency of phylogenetic studies conducted using various genomic regions.

With the markers used in the present study, the author clearly observed low species delimitation resolution. In many cases, some individuals from different species were clustered together even when the genomic regions were concatenated to improve the resolution. As mentioned above, in the genus Corydalis, the currently established taxonomy is mainly based on morphology and not on molecular genetic information (Oh, 1999; Oh et al., 2004; Oh et al., 2010). It is possible that the inconsistency noted between the previously established species delimitation and the phylogenies generated in the present study could be attributed to the fundamental difference between the morphological information and genetic information in their mechanisms for reflecting the inherent taxonomy of the target taxa. Therefore, although the observed discrepancy may lead to the conclusion that the taxonomy based on the phylogenies deviated from 
the "actual" taxonomy based on morphology, the author argue that this discrepancy should be more properly addressed and intensely explored to fully incorporate all the different observations available.

As the morphologies of Corydalis species are quite complicated, other tools, including phylogenetic analyses, are definitely needed for accurate identification of taxonomy for this genus. Indeed, in the previous studies, some taxonomical conclusions on Corydalis were successfully made by using both morphology and genetic analyses (Zhang et al., 2016; Xu and Wang, 2018). The author suggest that other genomic regions should be tested for their utilities in phylogenetic study of this genus or of section Corydalis, and that their molecular evolution should be studied in depth as well, so that the taxonomy of Corydalis can sufficiently benefit from molecular genetic information. Overall, the present study provides insights into the systematics of the Korean section Corydalis and demonstrates that chloroplast regions can be useful in phylogenetic analyses of this taxon when properly concatenated. The present study clearly suggests how to better utilize phylogenetic analyses and species delimitation in this intriguing and complicated taxon.

\section{ACKNOWLEDGMENTS}

This work was supported by a grant from the National Institute of Biological Resources (NIBR), funded by the Ministry of Environment (MOE) of the Republic of Korea (NIBR201907101).

\section{CONFLICTS OF INTEREST}

The authors declare no conflict of interest.

\section{REFERENCES}

Álvarez IJFW. and Wendel JF (2003). Ribosomal ITS sequences and plant phylogenetic inference. Mol. Phylogenet. Evol. 29: 417-434.

Amrine-Madsen H, Koepfli KP, Wayne RK, Springer MS (2003). A new phylogenetic marker, apolipoprotein B, provides compelling evidence for eutherian relationships. Mol. Phylogenet. Evol. 28: 225-240.

Bapteste E, Susko E, Leigh J, Ruiz-Trillo I, et al. (2008). Alternative methods for concatenation of core genes indicate a lack of resolution in deep nodes of the prokaryotic phylogeny. Mol. Biol. Evol. 25: 83-91.

Dong W, Liu J, Yu J, Wang L, et al. (2012). Highly variable chloroplast markers for evaluating plant phylogeny at low taxonomic levels and for DNA barcoding. PLoS One. 7: e35071.

Edgar RC (2004). MUSCLE: multiple sequence alignment with high accuracy and high throughput. Nucleic. Acids Res. 32: 1792-1797.

Fedde F (1936). Papaveraceae. Die Naturlichen Pflanzen-familien. 17: 5-145.

Hedges SB, Blair JE, Venturi ML, Shoe JL (2004). A molecular time scale of eukaryote evolution and the rise of complex multicellular life. BMC. Evol. Biol. 4:2.

Hooker JD and Thomson T (1855). Flora Indica 1: 121-272.

Jiang L, Li M, Zhao F, Chu S, et al. (2018). Molecular identification and taxonomic implication of herbal species in genus Corydalis (Papaveraceae). Molecules. 23: 1393.

Kolaczkowski B and Thornton JW (2004). Performance of maximum parsimony and likelihood phylogenetics when evolution is heterogeneous. Nature. 431: 980-984.

Komarov VL (1903). Florae Manshuriae 2. Act. Hort. Petrop. 22: 1-787.

Kress WJ, Wurdack KJ, Zimmer EA, Weigt LA, et al. (2005). Use of DNA barcodes to identify flowering plants. Proc. Natl. Acad. Sci. US A. 102: 8369-8374. 
Kubatko LS and Degnan JH (2007). Inconsistency of phylogenetic estimates from concatenated data under coalescence. Syst. Biol. 56: 17-24.

Kumar S, Stecher G, Tamura K (2016). MEGA7: molecular evolutionary genetics analysis version 7.0 for bigger datasets. Mol. Biol. Evol. 33: 1870-1874.

Li Q, Guo X, Niu J, Duojie D, et al. (2020). Molecular phylogeography and evolutionary history of the endemic species Corydalis hendersonii (Papaveraceae) on the Tibetan Plateau inferred from chloroplast DNA and ITS sequence variation. Front. Plant. Sci. 11: 436.

Lidén M, Fukuhara T, Axberg T (1995). Phylogeny of Corydalis, ITS and morphology. In Systematics and Evolution of the Ranunculiflorae (pp. 183-188). Springer, Vienna.

Lidén M (1996). New taxa of tuberous Corydalis (Fumariaceae). Willdenowia, 26: 23-35.

Lidén M, Fukuhara T, Rylander J, Oxelman B (1997). Phylogeny and classification of Fumariaceae, with emphasis on Dicentra sl, based on the plastid gene rps 16 intron. Plant. Syst. Evol. 206: 411-420.

Liu YY, Kan SL, Wang JL, Cao YN, et al. (2021). Complete chloroplast genome sequences of Corydalis edulis and Corydalis shensiana (Papaveraceae). Mitochondrial. DNA. B. Resour. 6: 257-258.

Mort ME, Archibald JK, Randle CP, Levsen ND, et al. (2007). Inferring phylogeny at low taxonomic levels: utility of rapidly evolving cpDNA and nuclear ITS loci. Am. J. Bot. 94: 173-183.

Mossel E and Vigoda E (2005). Phylogenetic MCMC algorithms are misleading on mixtures of trees. Science. 309: 2207-2209.

Nakai T (1909). Flora Koreana (I). J. Coll. Sci. Imp. Univ. Tokyo 26: 1-304.

Nakai T (1914). Plantæ novæ Japonicæ et Coreanæ 2. Bot. Mag. (Tokyo) 28: 28-29.

Nakai T (1952). A synoptical sketch of Korean flora. Bull. Natl. Sci. Mus., Tokyo 31: 1-152.

Oh BU (1986). A taxonomic study of genus Corydalis in Korea. Ph. D. thesis, Korea Univ. Seoul, Korea.

Oh BU (1999). A taxonomic review of Korean Corydalis (Fumariaceae). Korean J. Pl. Taxon. 29: 201-230.

Oh BU, Jang CG, Yoon CY (2004). Corydalis namdoensis BU Oh et JG Kin: A new species of Corydalis sect. Corydalis (Fumariaceae) from Korea. Korean J. Pl. Taxon. 34: 265-271.

Oh BU, Kim YY, Ji SJ, et al. (2010). Corydalis alata: A new species of Corydalis sect. Corydalis (Fumariaceae) from Korea. Korean J. Pl. Taxon. 40: 79-83.

Palibin J (1898). Conspectus flora Koreae, Par I. Acta. Hort.

Pérez-Gutiérrez MA, Romero-García AT, Fernández MC, Blanca G, et al. (2015). Evolutionary history of fumitories (subfamily Fumarioideae, Papaveraceae): An old story shaped by the main geological and climatic events in the Northern Hemisphere. Mol. Phylogenet. Evol. 88: 75-92.

Ren FM, Wang YW, Xu ZC, Li Y, et al. (2019). DNA Barcoding of Corydalis, the Most Taxonomically Complicated Genus of Papaveraceae. Ecol. Evol. 9: 1934-1945.

Rokas A, Williams BL, King N, Carroll SB. (2003). Genome scale approaches to resolving incongruence in molecular phylogenies. Nature. 425:798-804.

Shaw J, Lickey EB, Beck JT, Farmer SB, et al. (2005). The tortoise and the hare II: relative utility of 21 noncoding chloroplast DNA sequences for phylogenetic analysis. Am. J. Botany, 92: 142-166.

White TJ, Bruns T, Lee S, Taylor J (1990). Amplification and direct sequencing of fungal ribosomal RNA genes for phylogenetics. PCR protocols: a guide to methods and applications. 18: 315-322.

Wu J, Lin P, Guo Y, Liu M. (2020). The complete chloroplast genome of Corydalis conspersa. Mitochondrial. DNA. B. Resour. 5: 1977-1978.

Xu X and Wang D. (2018). Corydalis ternatifolia belongs to C. sect. Asterostigmata, not C. sect. Incisae (Papaveraceae): Evidence from morphological and phylogenetic study. Phytotaxa, 382: 193-203.

Xu X and Wang D (2021). Comparative chloroplast genomics of Corydalis Species (Papaveraceae): evolutionary perspectives on their unusual large scale rearrangements. Front. Plant. Sci. 11: 2243.

Yuan YM, Küpfer P, Doyle JJ (1996). Infrageneric phylogeny of the genus Gentiana (Gentianaceae) inferred from nucleotide sequences of the internal transcribed spacers (ITS) of nuclear ribosomal DNA. Am. J. Bot. 83: 641-652.

Zhang ML, Su ZY, Lidén M (2008). Corydalis DC. Flora of China, 7: 336-342.

Zhang ZX, Wang D, Yang X (2016). The taxonomic position of Corydalis parviflora Su \& Lidén (Papaveraceae), a genetically distinct species: Evidence from cpDNA and nDNA sequences. Biochem. Syst. Ecol. 67: 134-141. 